\title{
Conotruncal Heart Defects: Altered Tissue Morphology and Hemodynamics
}

\author{
Rebecca S Jones ${ }^{1,2}$, Lorain Junor ${ }^{2}$, Mary R Hutson ${ }^{3}$, Margaret L Kirby ${ }^{3,4}$, Richard L Goodwin ${ }^{5}$ \\ ${ }^{1}$ Biomedical Engineering Program, College of Engineering and Computing, University of South \\ Carolina, Columbia, SC 29208, USA \\ 2 Department of Cell Biology and Anatomy, University of South Carolina School of Medicine, \\ Columbia, SC 29209, USA \\ ${ }^{3}$ Department of Pediatrics (Neonatology), Neonatal-Perinatal Research Institute, Duke University \\ Medical Center, Durham, NC 297710, USA \\ ${ }^{4}$ Department of Cell Biology, Duke University Medical Center, Durham, NC 297710, USA \\ ${ }^{5}$ Department of Biomedical Sciences, University of South Carolina School of Medicine Greenville, \\ Greenville, SC 29605, USA
}

Each year in the US, about 40,000 babies are born with congenital heart defects (CHDs) [1]. While the array of causes of CHDs continues to widen and diversify, the prevalence of CHDs, especially the more common, less severe CHDs, is growing [2]. CHDs are known to be caused by genetic mutations and irregular hemodynamic forces during embryonic development; however, the cause of specific types of CHDs relative to these factors is much more obscure as many known aberrations in heart development produce a variable range of CHDs. Furthermore, the extent of the interaction between genetic and environmental components is an even more nebulous area of research [3]. To elucidate more information on the interplay between molecular and hemodynamic influences, we have utilized two different models of CHDs to analyze tissue morphology and create three-dimensional reconstructions of CHDs in order to predict hemodynamic properties. An avian model of Tetralogy of Fallot (TOF), characterized by ventricular septal defect, pulmonary obstruction (stenosis or atresia), overriding aorta, and right ventricular hypertrophy, has been developed through inhibition of Sonic hedgehog (Shh), which reduces proliferation of the secondary heart field (SHF). The Shh-null mouse was originally described as a phenotypic equivalent of TOF with pulmonary atresia as Shh is necessary for outflow tract (OFT), neural crest (NC) cell, and SHF proliferation. In previous studies, cyclopamine, an inhibitor of hedgehog signaling, decreased SHF proliferation and migration. Abnormal SHF proliferation has been linked to arterial pole defects, suggesting that cyclopamine treatment may yield arterial pole defects later in development [4]. Ablation of the NC has also been used as another avian model of CHDs that most commonly results in persistent truncus arteriosus (PTA) or other OFT malalignment defects such as double outlet right ventricle and TOF. NC ablation results in a failure of the SHF to contribute myocardium, which yields disrupted patterning of the arterial pole [4].

In the present study, cyclopamine-treated eggs were windowed at Hamburger Hamilton (HH) stage 14 to pipette $10 \mu \mathrm{L}$ of cyclopamine $(0.8 \mu \mathrm{g} / \mu \mathrm{L})$ or PBS/EtOH (control) onto the embryo, then resealed with tape and incubated. Cyclopamine treatment at $\mathrm{HH} 14$ coincides with the initial contribution of myocardium to the distal OFT by the SHF [4]. For neural crest-ablated eggs, a pulsed nitrogen/dye laser was used to ablate the NC cells from the midotic placode to the third somite of $\mathrm{HH}$ stage 8 chicken embryos. These cells seed the 3rd, 4th, and 6th pharyngeal arches and have been defined as the cardiac NC. At HH 42, eggs of both treatment types were harvested, and the hearts and outflow vessels were fixed in either paraformaldehyde or glutaraldehyde then paraffin-embedded, sectioned $(8 \mu \mathrm{m})$, and stained. H\&E staining was used to analyze overall morphology, and staining with aSMA, collagen type I, and DAPI was used for confocal microscopy (Figure 1). AMIRA software was used to generate 3D models 
of myocardial and vessel tissue (Figure 2, top row) in addition to flow space (Figure 2, bottom row) from tiled serial sections of the heart and associated vessels. Volumetric analysis was based on the AMIRA 3D rendering and input voxel size after z-plane alignment and positive staining selection for each image. Our results yielded a range of various cardiac malformations that showed differences in overall tissue structure and morphology, which would indicate aberrations in hemodynamic function.

\section{References:}

[1] MD Reller et al., The Journal of Pediatrics 153 (2008), p. 807.

[2] K Bjornard et al., Birth Defects Research Part A: Clinical and Molecular Teratology 97 (2013), p. 87.

[3] M Midgett, KL Thornburg, and S Rugonyi, American Journal of Physiology - Heart and Circulatory Physiology (2017).

[4] LA Dyer and ML Kirby, Developmental biology 330 (2009), p. 305.
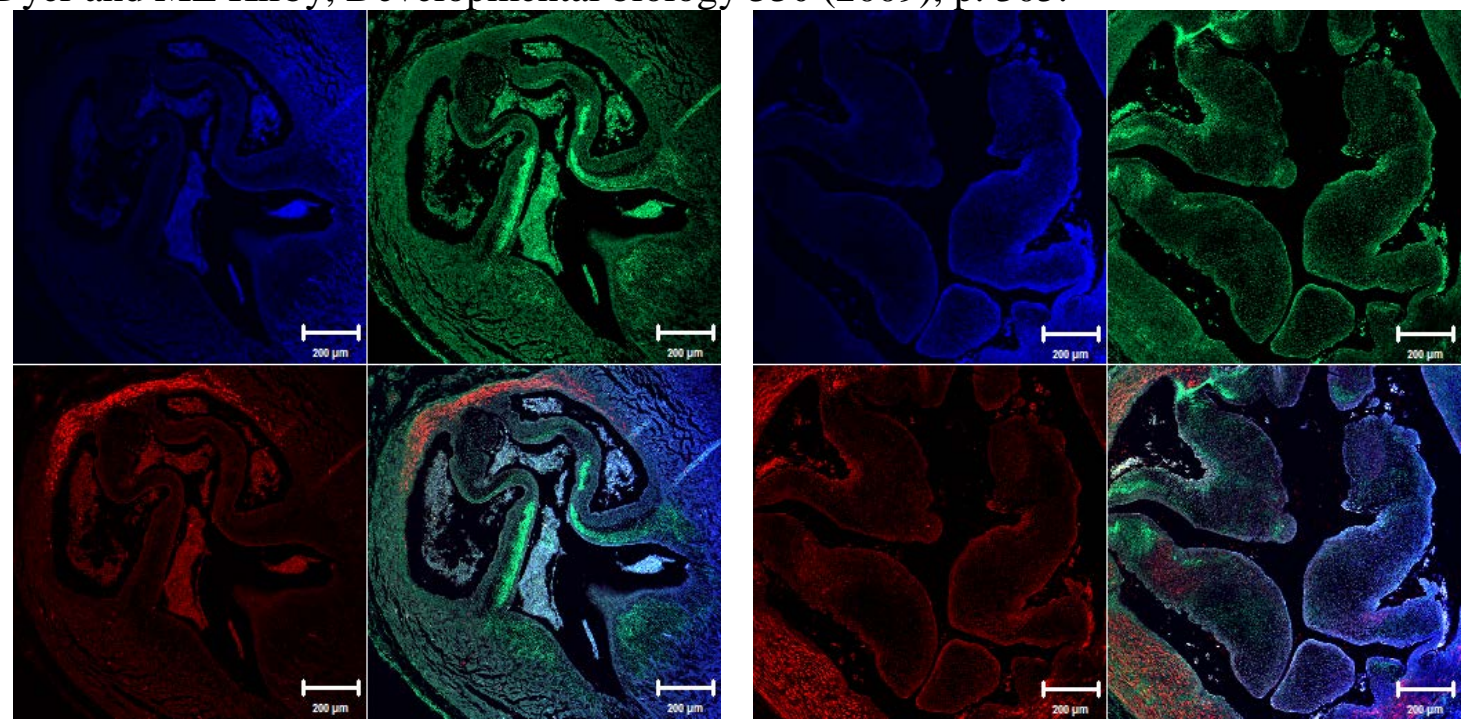

Figure 1. Structural Alterations in Valve Leaflets in Control (left) vs. Neural Crest Ablated (right) Hearts. HH 42 neural crest ablated hearts exhibit less collagen type I (green staining) in valve leaflets and more $\alpha$ SMA (red staining) at the leaflet connection to the vessel root than controls. Blue staining is DAPI.

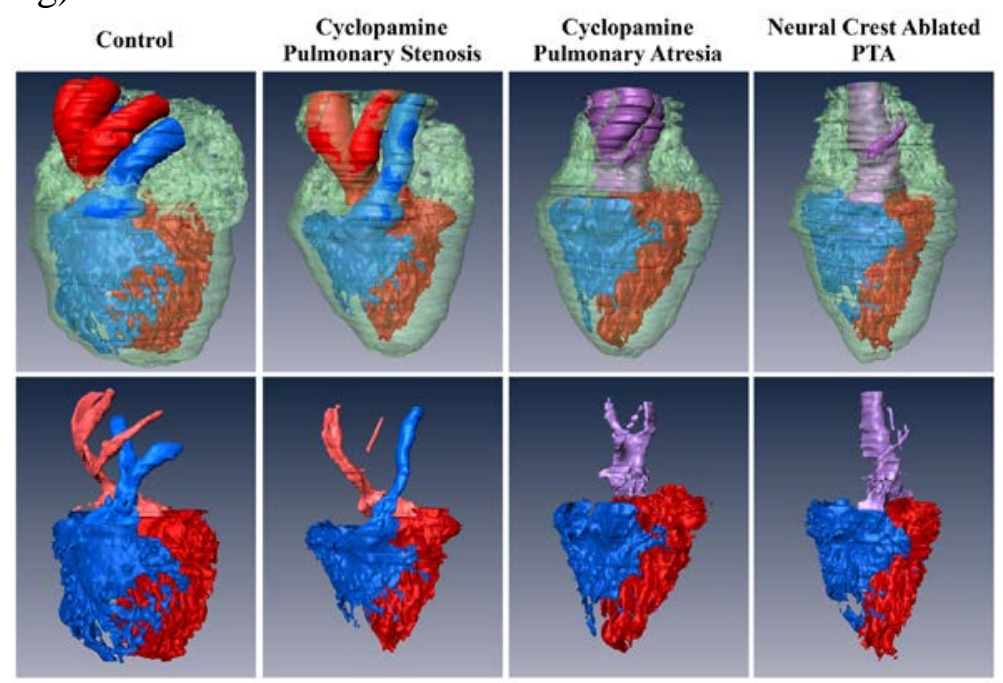

Figure 2. Amira 3D Reconstructions of HH42 Hearts (top row) and Flow Space (bottom row). Control, cyclopamine-treated, and neural crest ablated hearts exhibit cardiac malformations including pulmonary stenosis, pulmonary atresia, and PTA. 